\title{
Enhancing Line Capacity Utilization in Power Transmission System Using Active MVDC Link
}

\author{
Changhee Han ${ }^{1}$, Sungyoon Song ${ }^{1}{ }^{\mathbb{D}}$, Juyong Kim ${ }^{2}$ and Gilsoo Jang ${ }^{1, * \mathbb{C}}$ \\ 1 School of Electrical Engineering, Korea University, Anam-ro, Sungbuk-gu, Seoul 02841, Korea; \\ hch0806@korea.ac.kr (C.H.); blue6947@korea.ac.kr (S.S.) \\ 2 Korea Electric Power Corporation (KEPCO), Naju-si, Jeollanam-do 58322, Korea; juyong.kim@kepco.co.kr \\ * Correspondence: gjang@korea.ac.kr; Tel.: +82-3290-3426
}

Received: 25 February 2019; Accepted: 24 April 2019; Published: 26 April 2019

\begin{abstract}
As a proportion of generation using renewable energy increases in a power system, the need for facility investment is increasing in not only the distribution system but also the transmission system. However, it is inefficient to invest in new equipment to deal with the short peak output of renewable energy generation. For this reason, this paper proposes a method for operating the grid more flexibly, through an optimal operating strategy of the direct current (DC) line at medium-voltage (MV) level, thereby delaying the facility investment in the transmission line. In order to determine the optimal operating point of the converter connected with a DC line, the swarm intelligence-based optimization technique, which can minimize multi-objective function, is used. In addition, in order to overcome the communication dependency of the centralized control, which needs to receive information about the optimal operating point computed by the system operator, this paper proposes a decentralized emergency control (DEC) method in case of a communication fault.
\end{abstract}

Keywords: medium voltage direct current; multi-objective optimization; operation strategy; voltage source converter

\section{Introduction}

With a recent global effort to reduce carbon emissions, the share of renewable energy in electricity generation relative to coal or nuclear power generation is steadily increasing [1]. Due to the typical characteristic of renewable energy generation (REG) such as unstable output power fluctuation and unpredictability due to climate change, a variety of problems can arise in a power transmission and distribution system in which REG is highly penetrated. These problems include voltage fluctuation [2], load unbalancing [3], and reverse power flow [4]. Among these problems, this paper focuses on the inefficient use of line capacity due to REG. In areas with favorable climatic conditions for REG, there is a high demand for businesses using renewable energy. In such areas, the amount of generation by REG could be temporarily higher than the load demand, leading to reverse power flow to other areas. This ultimately comes back to the system operator with the burden of expansion of new facilities like transmission lines and substations. However, since a typical REG has a low utilization rate for the generation amount $[5,6]$, it is economically inefficient to add new facilities so as to meet the REG's temporary peak output power.

In order to overcome these inefficiencies, the application of a direct-current (DC) system at a medium voltage (MV) network has attracted attention. A medium voltage direct-current (MVDC) system refers to a DC system with a voltage level between high voltage direct-current (HVDC) and a low voltage direct-current (LVDC). With different purposes than HVDC, which is basically used for long-distance transmission, and LVDC, which is applied to microgrids, research on the use of MVDC is currently underway. The power flow through the alternating current (AC) line is passively 
determined by the line impedance, voltage magnitude and angle difference of buses. However, the power flow in the converter-based DC line can be manipulated by the operator. Therefore, research has been conducted to delay the grid expansion, increase the flexibility of the power system and operate the grid more efficiently by using the converter and DC link-based facility which is capable of power flow control [7-11]. In [7], research has been conducted into delays in the expansion of new facilities by connecting each distribution feeder with an MVDC link as the back-to-back (BTB) type in urban areas where the installation of a new line and transformer is difficult due to high load density and a complicated network. In addition, the application of an MVDC link at the normally open point (NOP) for use as soft open point (SOP) can be used to reduce power losses in the distribution network and balance loads between feeders [8-10]. There has also been a study focused on maximizing the hosting capacity of REG in distribution networks by converting the conventional AC line into a DC line so as to use the advantage of a voltage source converter (VSC) that can quickly change the output power [11].

The aforementioned studies have focused on improving the efficiency and flexibility of the radial distribution system using a controllable DC link. There is also research focused on the operation of the DC link that can contribute to the MV system or the transmission system which is meshed grid structure $[12,13]$. By connecting a region where REG is highly penetrated to another region which has a load pattern similar to REG using MVDC, the system operator can use the MVDC link to minimize the loss of the system and improve the line capacity utilization in the networks by calculating the optimal operation point [12]. However, this research has disadvantages to the extent that the data of the substations interconnected with another grid are always needed. There is also a study that suggests the operation of VSC-HVDC to minimize the transmission line loading [13], but this research focuses on using the VSC of high voltage and capacity level for improving the transmission system efficiency. Based on these studies, this paper focuses on the application of MVDC to increase the acceptability of REG in the transmission system and to delay the construction of new facilities by balancing the line capacity utilization in the networks. Balancing the line capacity utilization involves equalizing the current loading on each line in the network by forcing power flow from the line with high current loading to the line with low current loading. Also, the optimal operation process of the MVDC link which can minimize defined objective functions is proposed for more flexible and efficient gird operation. The objective functions representing the sum of line losses and line capacity utilization in the network are defined.

In order to determine the optimal operating point of the MVDC link that can minimize various objective functions, the swarm intelligence-based optimization technique is used [14-16]. Some studies have performed sensitivity analysis to determine the optimal dispatch of active and reactive power to minimize the objective functions desired by the grid operator [17-19]. However, this paper has focused on an optimization technique with an iterative process within given constraints to improve the accuracy in finding a global optimal point.

The day prior, an off-line study is carried out using the forecasted load and generation data. Through this off-line study, the optimal operating points of the MVDC link that minimize the objective function are determined every hour. While the MVDC link is operated by the optimal operating point as calculated a day prior, there are various ways in which the system operator can analyze the state of the network where the MVDC link is serving for the grid. The grid transformer (GT)-based control method presented in [12] is a method for measuring the amount of power flow in the grid-connected transformer and analyzing the difference between the total power generation and the load in the networks. This method is advantageous in that the structure of the communication system is simple. However, if the scale of the networks increases, it has a disadvantage in that it cannot detect the overload of the specific line in detail, because the GT-based control method only focuses on the difference between total amount of generation and load in networks. Therefore, this paper used a supervisory control and data acquisition (SCADA)-based control method to acquire data regarding the amount of generation and load, and the data of power flow in the networks observed by the system operator. In order to receive a command about the operating point of an MVDC link, a communication 
system is required between the system operator and the VSC station in the MVDC link. If a fault occurs in such a communication system and the VSC station no longer receives the information regarding the operating point sent by the system operator, the operating point of the MVDC link will remain constant over time. If the constant power is being transmitted by the DC line while neglecting the influence of the REG which fluctuates with time, output fluctuation of the REG can lead to overload on the transmission line or overvoltage. Therefore, it is particularly important to deal with communication failure when centralized control is performed in the network with a high penetration of REG [20,21].

To overcome dependence on the communication structure, there have been many studies on the decentralized control method in which VSC performs by itself for grid service without data communication at the central order [22,23]. In [22], a coordinated control scheme between VSCs to provide the most economical benefits to the AC/DC hybrid network has been proposed. There is also research that suggests an operation strategy of VSC by calculating the optimized voltage maintenance range at the connection point to minimize the total line losses when the VSC-based distributed generation (DG) is connected to the distribution network [23]. However, in all of these studies, the control is typically performed based on the voltage at the connection point because it is hard for VSC to detect the state of the entire grid in which it connected. In order to overcome this problem, this paper suggests a decentralized emergency control (DEC) method of the MVDC link as a measure against communication failure in VSC stations. Unlike other studies, this paper uses the difference of total power generation and load demand in the connected network with the MVDC link as an index to assess the state of the network. Based on the forecasted data of generation and load demand, the response curve, which is composed of the voltage angle of the connected bus and the amount of prior power reference in VSC station, is formulated to calculate the difference between the total power generation and the load demand in the network. Using this information, the VSC station by itself can calculate the operating point after the communication fault, reflecting the volatility of the REG.

The main contributions in this paper include: (1) suggesting an integrated operation strategy of an MVDC link that combines normal and emergency operation for the purpose that the MVDC link can contribute to the enhancement of operation efficiency in the transmission network, thereby delaying the construction of new facilities and allowing for further acceptance of REG. (2) Using a multi-objective optimization technique based on swarm intelligence to determine the optimal operating point of MVDC link for minimizing various objective functions with a new index which is used to equalize the line capacity utilization in networks. (3) Overcoming the communication dependency of the SCADA-based control method by proposing DEC method which reflects the volatility of the REG when a communication fault occurs in the VSC station of the MVDC link.

The rest of this paper is organized as follows: Section 2 describes the mathematical modeling of MVDC link and the objective functions for optimization. The basic principles of optimization technique are explained in Section 3. The proposed overall operation strategy of MVDC link is introduced in Section 4. In order to verify the effectiveness of the proposed method, case studies are conducted in Section 5 to compare the results after applying the method. Finally, the main conclusions of the paper are summarized in Section 6.

\section{Mathematical Modeling}

In order to determine the optimal operating point of the MVDC link, the power injection model of VSC is developed using the mathematical technique described herein. Then, the objective functions for assessing the effect of an MVDC link are formulated.

\subsection{Medium Voltage Direct-Current (MVDC) Link Modeling}

In this paper, mathematical modeling of MVDC link is conducted as a power injection model which consists of two VSCs as shown in Figure 1. If the power loss caused by switching devices or dc capacitor is quite low as compared to the power output of VSC, it could be omitted [12]. 


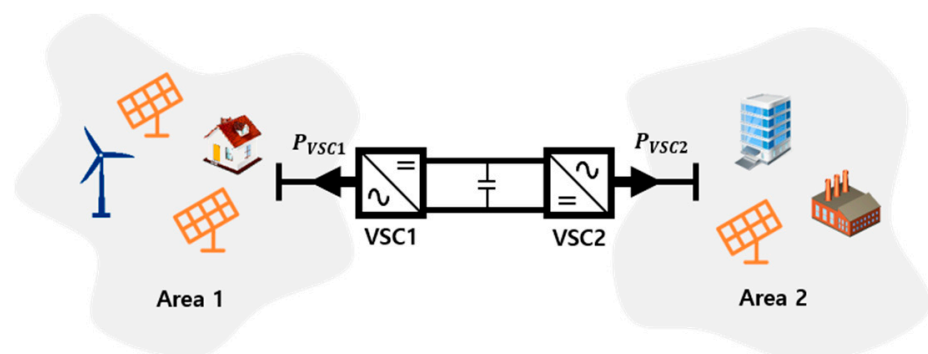

Figure 1. Connection of two networks using medium voltage direct-current (MVDC) link.

Because the amount of active power output of the one VSC is equal to the absorbed active power of the other VSC, the relationship of active power from each VSC could be represented as (1). $P_{V S C_{1}}$ and $P_{V S C_{2}}$ represent the amount of active power injected into the connected networks from each VSC.

$$
\begin{gathered}
P_{V S C_{1}}+P_{V S C_{2}}=0 \\
\sqrt{\left(P_{V S C_{k}}\right)^{2}+\left(Q_{V S C_{k}}\right)^{2}} \leq S_{V S C_{k}}
\end{gathered}
$$

Equation (2) refers to the maximum active and reactive power output of VSC that are limited by the $k$ th converter's capacity, $S_{V S C_{k}} . P_{V S C_{k}}$ and $Q_{V S C_{k}}$ is the active and reactive power output of $k$ th VSC, respectively.

$$
Q_{\min _{k}} \leq Q_{V S C_{k}} \leq Q_{\max _{k}}
$$

$Q_{\text {min }_{k}}$ is the lower limit and $Q_{\max _{k}}$ are the upper limit of reactive power output by $k$ th VSC. The reactive power output of each VSC is used to set the voltage magnitude at the point of common coupling (PCC) into a stable range. In this paper, the amount of reactive power reference of VSC is automatically determined so as to maintain the AC voltage magnitude of PCC as a set point (1 p.u).

\subsection{Objective Function Modeling}

The objective functions for optimal operation of the MVDC link $\left(f_{o b j}\right)$ can be varied according to the network operator's purpose. In this paper, three kinds of objective functions are defined. The controllable output of active power in the MVDC link $\left(P_{r e f}\right)$ is used as a decision variable for minimizing the objective function, which is shown in (4).

$$
\begin{gathered}
\min _{P_{r e f}} f_{o b j} \\
f_{o b j}=\left\{f_{\text {loss }}, f_{L C U}, f_{D E V}\right\}
\end{gathered}
$$

The three objective functions defined as (5) in this paper are as follows: sum of line losses in the network, line capacity utilization ratio, and deviation of line utilization. The objective function to minimize line loss is formulated as (6). $I_{i}$ is the line current flowing at the $i$ th line with resistance $r_{i}$. The total number of lines in the network is represented as $n_{l}$.

$$
\begin{gathered}
f_{\text {loss }}=\sum_{i=1}^{n_{l}} I_{i}^{2} \cdot r_{i} \\
f_{L C U}=\sum_{i=1}^{n_{l}}\left(\frac{S_{i}}{S_{i, \text { rate }}}\right)^{2} \\
f_{D E V}=\sqrt{\frac{1}{n_{l}} \sum_{i=1}^{n_{l}}\left(\frac{S_{i}}{S_{i, \text { rate }}}-\frac{\sum_{i=1}^{n_{l}} S_{i}}{n_{l}}\right)^{2}}
\end{gathered}
$$


Equation (7) is an index indicating the total line loading in the network. $S_{i}$ means the apparent power flow of the $i$ th line which has the rated power capacity of $S_{i, \text { rate }}$. Equation (8) means the degree of how uniformly the line utilization is distributed in the network. It represents the deviation of the line current loading in the network. The minimization of $f_{L C U}$ and $f_{D E V}$ means maintaining the utilization of each line balanced within the network to prevent the power flow from being overloaded to a specific line, which in turn improves the flexibility in the network.

\subsection{Networks Constraints Modeling}

There are two constraints used in the proposed optimization process: device constraint and network constraint. Device constraint means a limited power capacity of VSC devices, which is represented as Equations (2) and (3).

$$
\begin{gathered}
V_{\min } \leq V_{k} \leq V_{\max }, k \in\left\{1,2, \cdots, n_{B}\right\} \\
S_{i} \leq S_{i, \max }, i \in\left\{1,2, \cdots, n_{l}\right\}
\end{gathered}
$$

Network constraint includes a permitted range of voltage magnitude for stability of networks and a maximum capacity of transmission line. The network constraints are shown in Equations (9) and (10). $V_{\min }$ and $V_{\max }$ represent the lower and upper bounds of $k$ th bus voltage $\left(V_{k}\right)$, respectively. $S_{i, \max }$ is the maximum apparent power of the $i$ th branch. $n_{B}$ means the total number of buses in the network.

\section{Optimization Algorithm}

In this paper, the optimization algorithm based on intelligence of swarm is used to calculate the optimal operating point of the MVDC link, which can minimize single or multiple objective functions as defined by network operator.

\subsection{Particle Swarm Optimization (PSO) Algorithm}

The particle swarm optimization (PSO) algorithm is an optimization technique using a swarm intelligence, inspired by social behavior of bird flocking [15]. With a population of randomly scattered candidate solutions called 'particles', the search is conducted by repeatedly moving particles in search space according to a mathematical formula about particle's position and velocity. While a particle is moving, the velocity of each particle is calculated to include three characteristics: Inertia, Cognitive, and Social parts. The Inertia part means the property of a particle that keeps the previous trajectory. The Cognitive part accounts for the tendency to return to the particle's own best positions that were the best positions of the particle itself among their previously iterative path. Lastly, the Social part stands for the particle's movement to the global optimum solution. $x_{n}(t)$ in Equations (11) and (12) means the $n$th particle's position at iteration step $t . v_{n}(t+1)$ is the velocity of the particle, which is shown in (12). $n_{P}$ is the total number of particles.

$$
\begin{gathered}
x_{n}(t+1)=x_{n}(t)+v_{n}(t+1), n \in\left\{1,2, \cdots, n_{P}\right\} \\
v_{n}(t+1)=w \cdot v_{n}(t)+r_{1} \cdot c_{1} \cdot\left(p_{n}(t)-x_{n}(t)\right)+r_{2} \cdot c_{2} \cdot\left(g(t)-x_{n}(t)\right)
\end{gathered}
$$

In (12), the velocity of a particle consists of the sum of three terms. The first represents the Inertia part, the second is the Cognitive part, and the last term is the social part. $p_{n}(t)$ is the best positions of the particle itself among its previously iterative path, and $g(t)$ is the global optimum positions (called 'leader') at iterative step t. $w, c_{1}$ and $c_{2}$ means the weight factors of the three characteristic parts in the velocity. $r_{1}$ and $r_{2}$ are real numbers randomly generated between 0 and 1 so that a particle can have stochastic natures in 'cognitive' and 'social' parts. 


\subsection{Multi-Objective Optimization Using PSO Algorithm}

In the case of performing optimization for more than one objective function, it can be implemented by modifying the process of the existing PSO algorithm [16]. In the multi-objective optimization process, it is defined that solution $x_{1}$ 'dominates' the other solution $x_{2}$ if the following two conditions are satisfied. This is shown in Equations (13) and (14).

$$
\begin{aligned}
& \text { for all } i \in\left\{1,2, \cdots, n_{o b j}\right\}, f_{i}\left(x_{1}\right) \leq f_{i}\left(x_{2}\right) \\
& \text { for any } j \in\left\{1,2, \cdots, n_{o b j}\right\}, f_{j}\left(x_{1}\right)<f_{j}\left(x_{2}\right)
\end{aligned}
$$

$n_{o b j}$ means the number of defined objective functions. If solution $x_{1}$ dominates $x_{2}, x_{1}$ is defined as the 'non-dominated' solution. After performing the optimization process for searching non-dominated solutions, a set of non-dominated solutions within the entire search area is called 'Pareto optimal front' (POF). POF means that an improvement of one objective function can reach some area that leads to deterioration of the other objective function [24]. The overall sequence of the PSO algorithm for the multi-objective optimization is as follows:

(1) A population of initial particles is randomly generated. Particles are composed of decision variables for optimization.

(2) Objective functions are calculated using the information of initial particles. Then a set of particles which have non-dominated solutions is determined and the leader particle is selected.

(3) The velocity of each particle is calculated by (12), and the particles move along the calculated velocity.

(4) To prevent particles from gathering in a local optimal point, arbitrary particles are selected and their positions are changed to generate mutations.

(5) Objective functions are calculated and the evaluation process to determine the non-dominated solution is conducted including mutations and existing particles.

(6) The information of the non-dominated particles is collected by repeating the movement of particles and generation of the mutation.

(7) After the iteration, POF is constructed with non-dominated solutions and the operator selects a compromise solution from these.

\section{Process for Operating MVDC Link}

This paper divided the operation of the MVDC link into two stages: the off-line study stage and the on-line operation stage. In the off-line study stage, the system operator should perform two analyzes as shown in Figure 2. The first is to calculate the optimal operating points of the next day through the PSO algorithm, and the second is the data build-up process that calculates the response curves for the emergency control to prepare for the communication failure. After the off-line study, the MVDC link at the on-line operation stage receives information about the operating points every hour, and continually checks whether the communication failure occurs.

\subsection{Process of Determining Daily Optimal Operating Point}

In the off-line study performed a day prior, based on the forecasted data regarding the amount of REG and load, the hourly optimal operating points of the MVDC link of the next day are determined for minimizing the objective functions through the PSO algorithm.

The flowchart in the blue frame in Figure 2 shows the process of the PSO algorithm for determining the optimal operating points of the MVDC link when there are two or more objective functions. In the on-line operation stage, the MVDC link receives the information for the optimal operating point $\left(P_{r e f}\right)$ every time from the centralized control center. 
Since the particles move only within the range satisfying the network constraint conditions shown in (9) and (10), when the calculated optimum operating point of the MVDC link is applied the problem such as line overload or overvoltage does not occur.

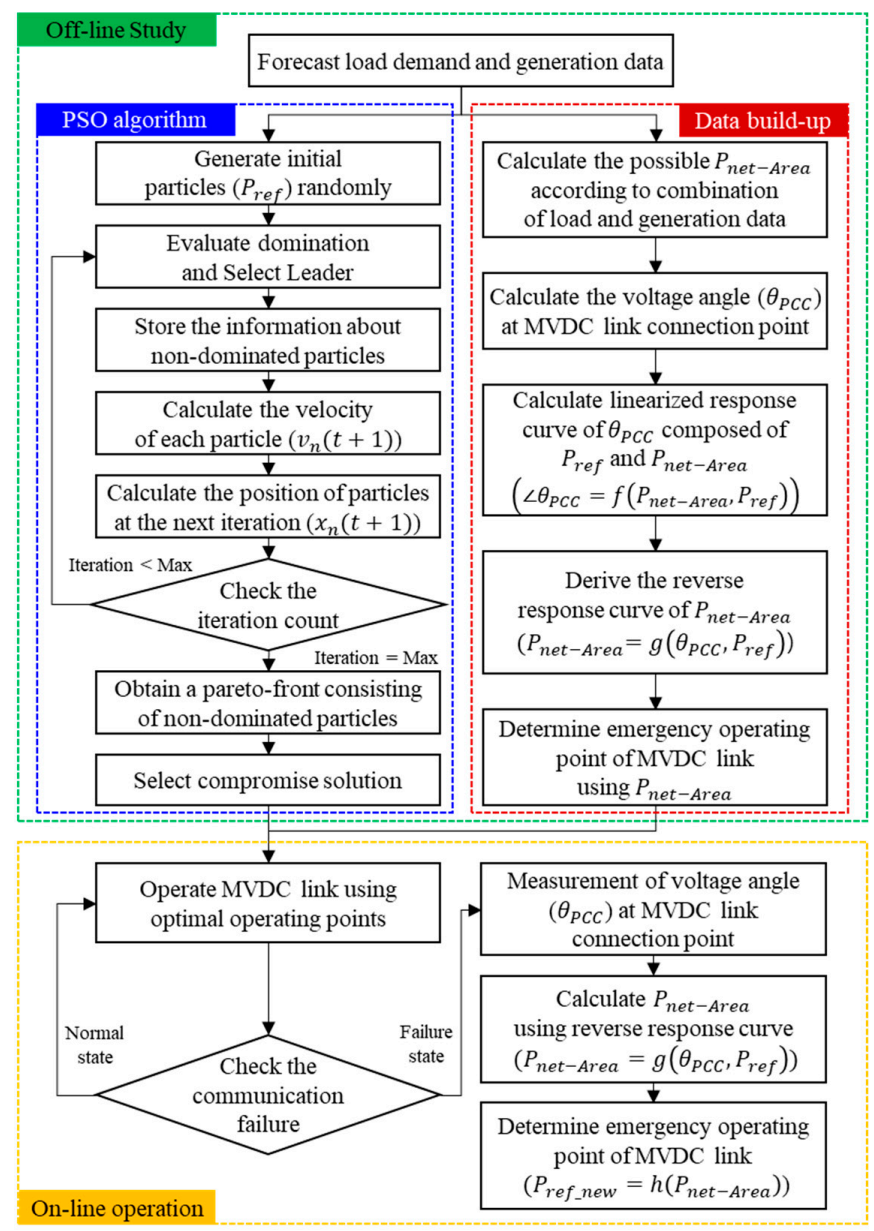

Figure 2. Flowchart for operating the MVDC link.

\subsection{Proposed Decentralized Emergency Control Method}

As shown in the yellow frame in Figure 2, the MVDC link receives the information about the optimal operating point from the network operator every hour. If a fault occurs in the communication system during the on-line operation, it is impossible to obtain the $P_{r e f}$ information for the next time step. In order to overcome this problem, this paper proposes an algorithm that estimates the total amount of generation and load demand in the connected networks with the MVDC link by using information that can be directly received from the VSC station. Using the estimated total amount of generation and load demand, the MVDC link can determine the operating point by itself in the case of a communication failure.

As illustrated in Figure 2 with the red frame, the data build-up process is needed to derive a response curve for the relationship between the information that can be directly received at the VSC station and the indicator representing the network status. If it is assumed that REG in the network generates with a fixed power factor and the VSC of the MVDC link automatically determines the reactive power output for $\mathrm{AC}$ voltage control, the voltage magnitude and angle at each bus in the network are determined by the changeable active power sources. 
The changeable active power sources mean the REG, load, and active power reference of MVDC link $\left(P_{r e f}\right)$. This is formulated as (15) and (16).

$$
\begin{gathered}
P_{\text {net-Area }}=\sum_{i=1}^{n_{G}} P_{G(i)}-\sum_{j=1}^{n_{L}} P_{L(j)} \\
V_{k} \angle \theta_{k}=f\left(P_{\text {net-Area }}, P_{\text {ref }}\right), k \in\left\{1,2, \cdots, n_{B}\right\}
\end{gathered}
$$

$P_{\text {net-Area }}$ refers to the difference between total active power generation and load demand in a specific area, and $V_{k} \angle \theta_{k}$ is the magnitude of voltage and angle at $k$ th bus in that area. $n_{G}$ and $n_{L}$ mean the total number of generators and loads in the area, respectively. $P_{G(i)}$ is the amount of active power generation from $i$ th generator, and $P_{L(j)}$ represents the amount of active power consumption from jth load.

Since the variation of generation and load demand is reflected in $P_{\text {net-Area }}$, the voltage magnitude and angle at any bus in the area can be expressed as (16). $f(a, b)$ denotes the result of power flow calculation which can be vary depending on $a$ and $b$. At the PCC connected with the MVDC link, the voltage magnitude can be assumed to be constant through the AC voltage control of the VSC. Since the magnitude of the voltage at the PCC is 1.0 p.u, (16) can be expressed as (17) at PCC only.

$$
1.0 \angle \theta_{P C C}=f\left(P_{\text {net-Area }}, P_{\text {ref }}\right)
$$

Through this, the voltage angle at the PCC is only determined by the combination of $P_{\text {net-Area }}$ and $P_{\text {ref }}$. After performing a number of simulations within the range of possible combinations of $P_{\text {net-Area }}$ and $P_{r e f}$, it is possible to derive a reverse relation like (18) for $P_{\text {net-Area }}$.

$$
P_{\text {net-Area }}=g\left(\theta_{P C C}, P_{r e f}\right)
$$

If the data represented as (17) and (18) are built up through the off-line study, the information about $P_{\text {ref }}$ and $\theta_{P C C}$ can be used to derive $P_{\text {net-Area }}$. This means that using the measured $\theta_{P C C}$ and $P_{\text {ref }}$, the VSC station can calculate $P_{\text {net-Area }}$ by itself based on (18). In this case, the optimal operating point through PSO algorithm cannot be calculated, because $P_{\text {net-Area }}$ only indicates the total difference of generation and load, which does not reflect detailed information about the respective amount of generation and load.

If a response curve for determining a new operation point using $P_{\text {net-Area }}$ as an input variable is calculated in advance by an off-line study, the VSC station can determine the new operating point $\left(P_{\text {ref_DEC }}\right)$ as shown in (19). In this paper, the relationship between the amount of power flow in the AC line at the MVDC link installation location and $P_{\text {net-Area }}$ is taken as the response curve.

$$
P_{\text {ref_DEC }}=h\left(P_{\text {net-Area }}\right)
$$

Through the proposed emergency control method, it is possible to switch to DEC mode in the case of a communication error. Since the emergency operating point is not an optimized value by off-line study, it may have a lower effect on the grid in terms of network efficiency. However, in this paper, we focus on the advantage of decentralized control, which does not require information reception at the central control center. The entire process of allowing the VSC calculate $P_{\text {net-Area }}$ by itself can be summarized as follows:

(1) Calculate the possible combination of $P_{\text {net-Area }}$ using the power amount of each generator and load during a day. It is made by uniformly changing the load demand and power generation level from $0 \%$ to $100 \%$, respectively.

(2) A large number of scenarios are created by changing the $P_{\text {ref }}$ from $-100 \%$ to $100 \%$ within the operating range for each $P_{\text {net-Area }}$. 
(3) Power flow calculation is performed for all scenarios. At this time, it is assumed that each generator and load have a fixed power factor and that the reactive power output of the MVDC link is determined for AC voltage control.

(4) Record the magnitude of the voltage angle at the PCC $\left(\theta_{P C C}\right)$ obtained as a result of the power flow calculation for all scenarios.

(5) Using the recorded data, a large number of power flow calculation results are placed in the three-dimensional plot of the $\mathrm{x}_{-}, \mathrm{y}-$, and z-axis, which means $P_{\text {ref }}, P_{\text {net-Area }}$, and $\theta_{P C C}$, respectively.

(6) Then using the curve fitting progress [25], it is possible to form an approximated surface and derive a linearized response curve shown in Equation (17).

(7) Another response curve which is shown in Equation (18) is derived by re-organizing the existing response curve against $P_{\text {net-Area }}$.

(8) When operating as DEC mode, $\theta_{P C C}$ observed by the VSC and $P_{\text {ref }}$ are substituted into the response curve to calculate the $P_{\text {net-Area }}$.

(9) Using the calculated $P_{\text {net-Area }}$, VSC determines by itself a new operating point.

In this paper, the curve fitting progress was performed using the Curve Fitting Toolbox provided by MATLAB software program [25].

\subsection{Operation Strategy under Uncertainty}

Through the proposed process, optimal operating points of the MVDC link are pre-calculated through an off-line study stage performed on the previous day using the forecasted load demand and generation data. However, since the predicted data may not correspond with the actually observed data at the On-line operation stage, the system operator should use the SCADA system to continually check whether the predicted value in the area matches the actual measured value.

If the measured amount of total load demand and generation in the area in which the MVDC link is connected are significantly different from the forecasted value, the existing operating point of the MVDC link is no longer the optimal operating point, which reduces the efficiency of the proposed method. In this case, the optimal operating point of the MVDC link should be newly calculated. However, since the proposed optimization method by using the PSO requires a large number of power flow calculations to be repeated, it is difficult to perform the optimization process in real time because of a long computing time.

As a solution for this, the optimal operating points of the MVDC link can be pre-calculated for all scenarios that can be generated by using possible combinations of the load demand and power generation amount. After calculating the optimal operating points of the MVDC link for all possible scenarios, these are constructed in lookup table form, in which the optimal operating points are represented as the matched values for different combination of total load demand and power generation in the area. Using this strategy, if there is a difference between the forecasted data and the measurement value during the On-line operation, the system operator can use the lookup table to select another optimal operating point of the MVDC link that matches to the measured total load demand and generation amount. This operating strategy allows the system operator to change the optimal operating point of the MVDC link flexibly in response to uncertainties in load demand and REG output.

\section{Case Studies}

In order to evaluate the proposed method, case studies were carried out using the real network data of the Korean power system. The simulations were conducted using PSS ${ }^{(r)}$ E program (Power Transmission System Planning Software). Due to the national energy policy, which mandates more renewable energy resources, the amount of REG in the Korea power system in 2031 will be about 50 GW, which would account for $20 \%$ of the total power generation capacity in Korea [26,27]. 
In this paper, the test network was determined as the 'Jeon-Nam' area in the Korea power system, because the planned REG in 2031 at this area is the largest in Korea because of its geographical and weather characteristic condition [27]. In the 'Jeon-Nam' area, two candidates for MVDC link installation were proposed as shown in Figure 3.

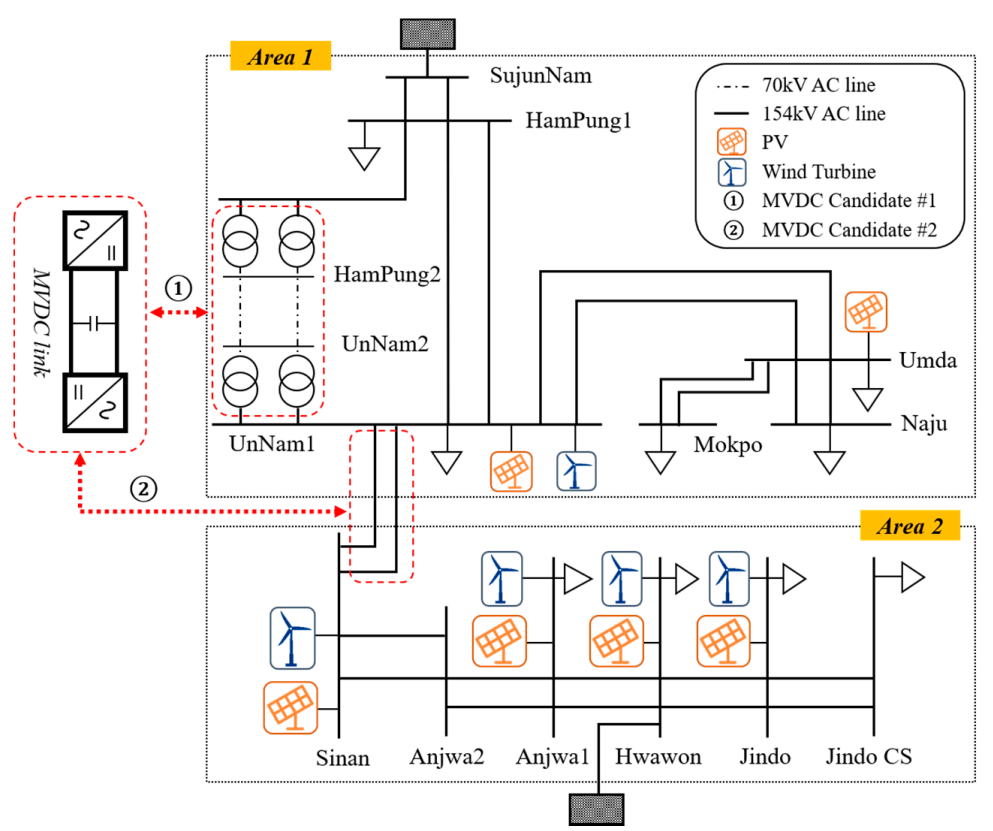

Figure 3. Representation of MVDC link candidates in the Korean power system.

The first candidate for MVDC link installation was proposed as an alternative to a 70-154 kV substation and line which connects 'HamPung1' and 'UnNam1'. The purpose of this MVDC link is to increase the efficiency of the line capacity utilization in the network by forcing the power flow through the DC line that previously did not flow due to the high reactance of the transformer when the line between two substations was an AC line.

The second candidate is located between 'Sinan' and 'UnNam1'. The purpose of this MVDC link is to minimize line loss and to overcome the overvoltage or overload problems that could arise when a large amount of REG is installed in the networks.

Also, in this paper, since the test network denoted by Area 1 and 2 in Figure 3 represents a specific part of the South Korea's national power transmission system, this test system is connected to a large and robust network that is another part of the national power transmission network. Therefore, in Figure 3, it can be assumed that another robust large-scale power system is connected to the upper side of the 'SujunNam' bus in Area 1 and the lower side of the 'Hwawon' bus in Area 2, respectively. This means that the case studies in this paper assume that the difference in load demand and power generation of Area 1 and 2 is supplied from the other large-scale power systems each connected to 'SujunNam' and 'Hwawon' buses.

\subsection{MVDC Application for Enhancing Line Utilization}

In the Korean power system in 2031, new substations and transmission lines connecting 'UnNam' and 'HamPung' will be installed to accept additional REG. Despite the fact that the substation and line will be newly constructed with the purpose of accommodating additional REG, the high reactance of the transformer in the substation makes the efficiency of line capacity utilization low. In addition, since REG has high output variability over time, it is inefficient to install new facilities considering the peak generation amount of REG. In order to overcome this challenge in AC grid expansion, this paper suggests the application of MVDC link as an alternative to a substation and AC line as shown in Figure 4. 


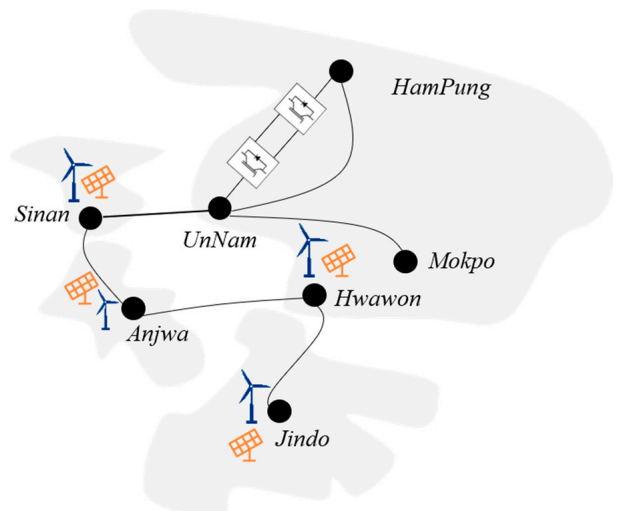

Figure 4. MVDC link candidate \#1. HamPung-UnNam connection.

In order to conduct an off-line study, the one-day profiles of total load demand and generation in the Area 1 are used to perform an optimization process. The daily profiles are shown in Figure 5. Table A2 in Appendix A shows the maximum amount of generation by REG and load demand assumed in this simulation.

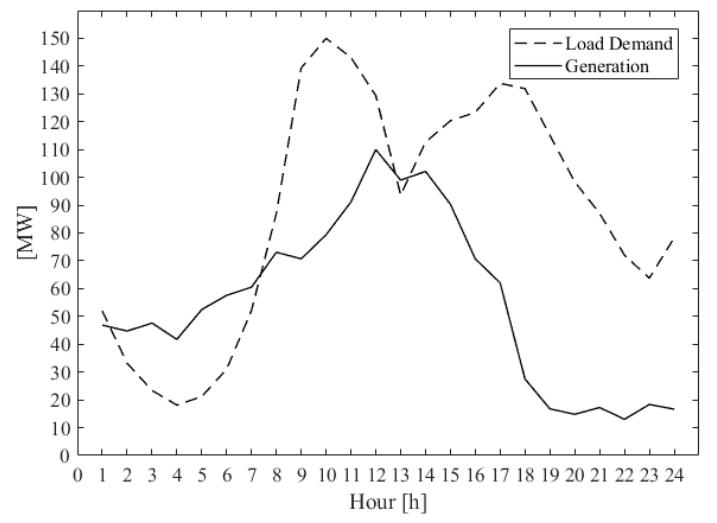

Figure 5. Daily profiles of total load and generation in Area 1.

Since the main purpose of the MVDC link in this case is to balance the utilization of line capacity in Area 1, the objective functions are composed of line capacity utilization $\left(f_{L C U}\right)$ and deviation $\left(f_{D E V}\right)$ index. Using the forecasted daily data of the next day, the optimal operating point of the MVDC link is calculated every hour to minimize the multi-objective functions. The maximum capacity of the MVDC link is assumed to be 100 MVA.

Figure 6 shows the trajectory of moving particles to minimize the two objective functions in the multi-objective PSO algorithm. Figure 7 shows the POF for two objective functions at a specific time $($ Hour $=13 \mathrm{~h})$ in the process of determining the optimal operating point of the MVDC link in Area 1 over time.

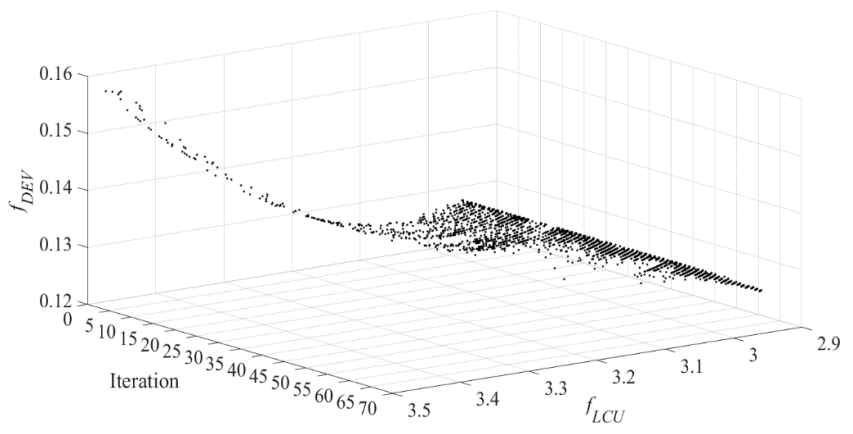

Figure 6. Trajectory of particles for minimizing multi-objective functions. 


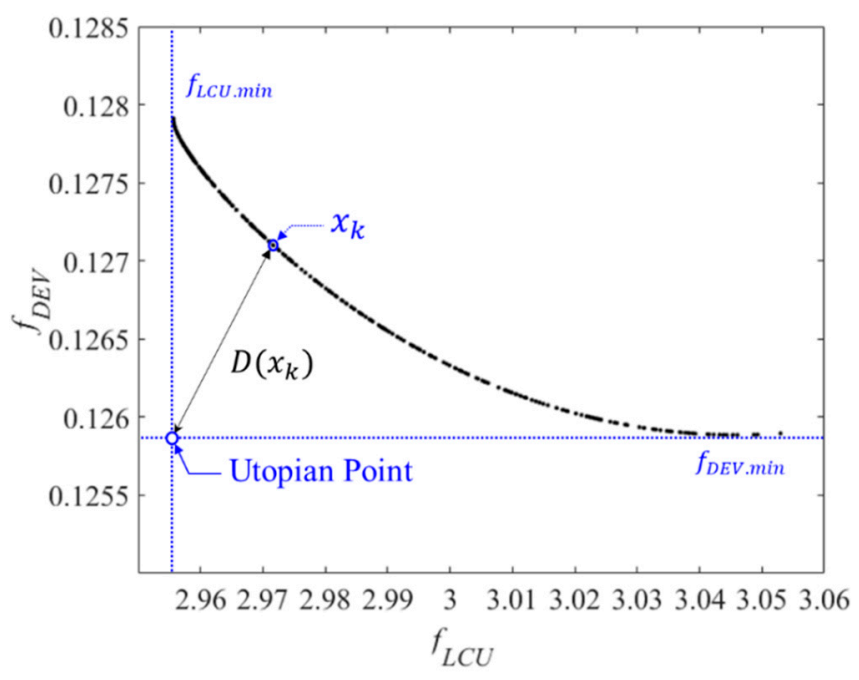

Figure 7. Pareto optimal front (POF) of the multi-objective function.

In order to determine a compromise solution, the Utopian point method is used in this paper [28]. This method is widely used for determining compromise solution when there is no preference information between the two objective functions. The utopian point means the ideal point which consists of the minimum value of each objective function in the POF. The compromise solution $\left(x_{\text {opt }}\right)$ is determined by the point with the shortest distance $\left(D\left(x_{k}\right)\right)$ from the utopian point among the points $\left(x_{k}\right)$ on the POF Equations (20) and (21). $f_{L C U, \min }$ and $f_{D E V, \min }$ represent minimum value of objective functions on the POF, respectively. $n_{\text {opt }}$ is the total number of particles on the POF.

$$
\begin{gathered}
D\left(x_{k}\right)=\sqrt{\left(f_{L C U, \min }-f_{L C U}\left(x_{k}\right)\right)^{2}+\left(f_{D E V, \text { min }}-f_{D E V}\left(x_{k}\right)\right)^{2}} \\
D\left(x_{\text {opt }}\right)=\min D\left(x_{k}\right), k \in\left\{1,2, \cdots, n_{\text {opt }}\right\}
\end{gathered}
$$

Figure 8 represents the result of calculating the hourly optimal operating points of MVDC link $\left(P_{\text {opt }}\right)$ in Area 1, reflecting the load and power generation in Figure 5. The operating points for each hour refer to compromise solutions to minimize both of the objective functions. The positive sign of $P_{\text {opt }}$ means that the MVDC link should transfer active power from 'UnNam1' to 'HamPung1'.

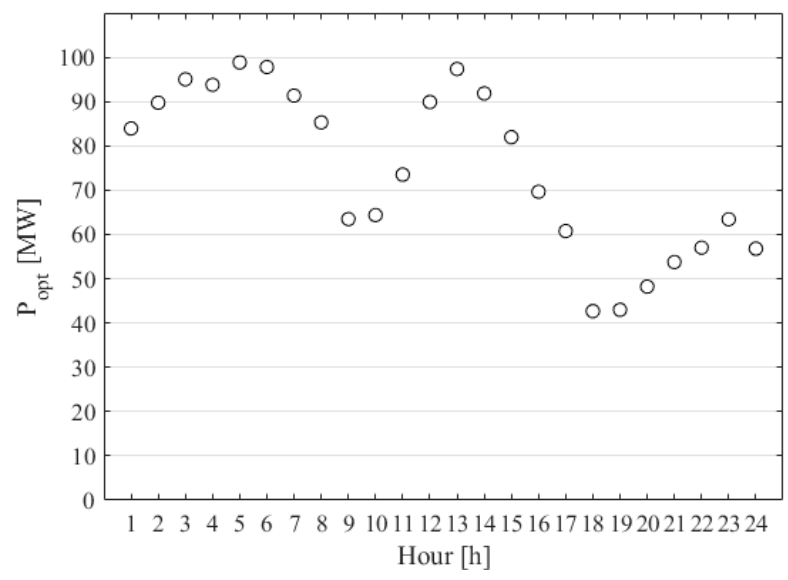

Figure 8. Optimal operating point of MVDC link candidate \#1.

Figure 9 shows the effect on the deviation index $\left(f_{D E V}\right)$ and total line capacity utilization index $\left(f_{L C U}\right)$ when the MVDC link is applied. To verify the superiority of the proposed method, we compared the two objective functions when the MVDC link is operated in the different method [29]. In [29], 
VSC is operated as the fixed active power output setpoint over time. The comparison between the fixed active power output of 80MW and the proposed optimal operation was performed. As shown in Figure 9, by applying the MVDC link to Area 1, the two objective functions are reduced during the day. Especially, it is more effective to reduce the objective functions when operating with the proposed optimal operating method than when the MVDC link is operated with fixed output. The average value of $f_{D E V}$ per day is reduced by about $24 \%$, and $f_{L C U}$ is reduced by about $42 \%$. This means that by forcing power flow in the specific line through the MVDC link, it is possible to avoid the problem of power flow being driven to some specific line. Further, this allows the networks to have the flexibility to deal with fluctuation of REG and load demand.

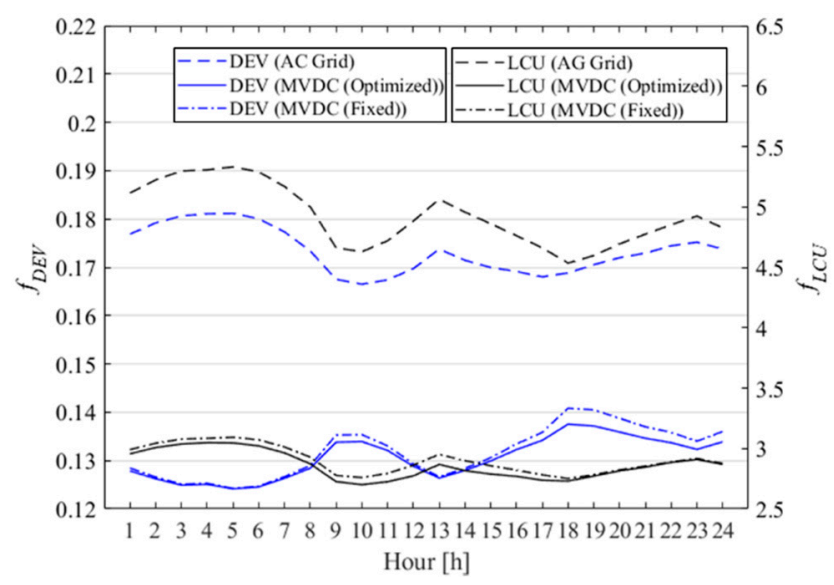

Figure 9. Comparison of two objective functions in Area 1.

Figure 10 shows a comparison of the line utilization rate of each line at a specific time (Hour $=1 \mathrm{~h}$ ) in Area 1. The amount of power flow by MVDC link in Figure 10 is about $83.9 \mathrm{MW}$ in that time. We can see in Figure 10 that the utilization rate of each line in Area 1 is equalized. The information about the branch number in Figure 10 is provided by Table A1 in Appendix A.

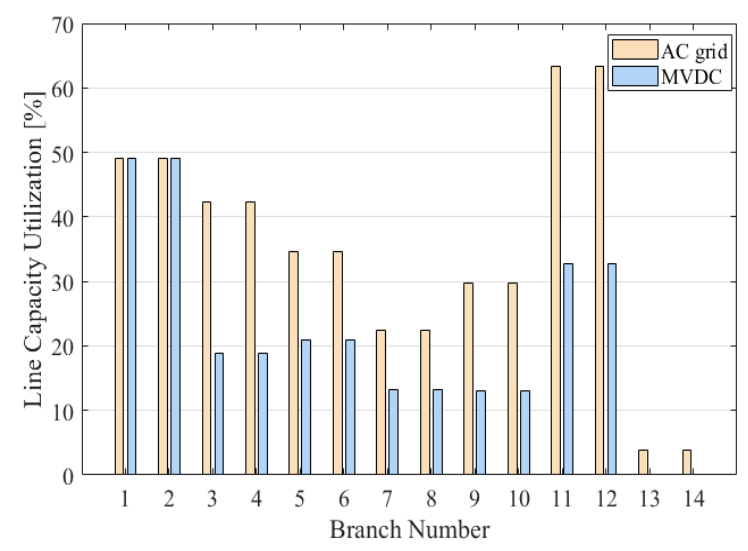

Figure 10. Comparison of line capacity utilization in Area 1.

\subsection{MVDC Application for Line Loss Minimization}

The second candidate for the MVDC link is proposed as an alternative to the AC line connecting Area 2 and 1 . The Area 2 is the terminal network with a high proportion of REG, and Area 1 is the mainland network as shown in Figure 11. 


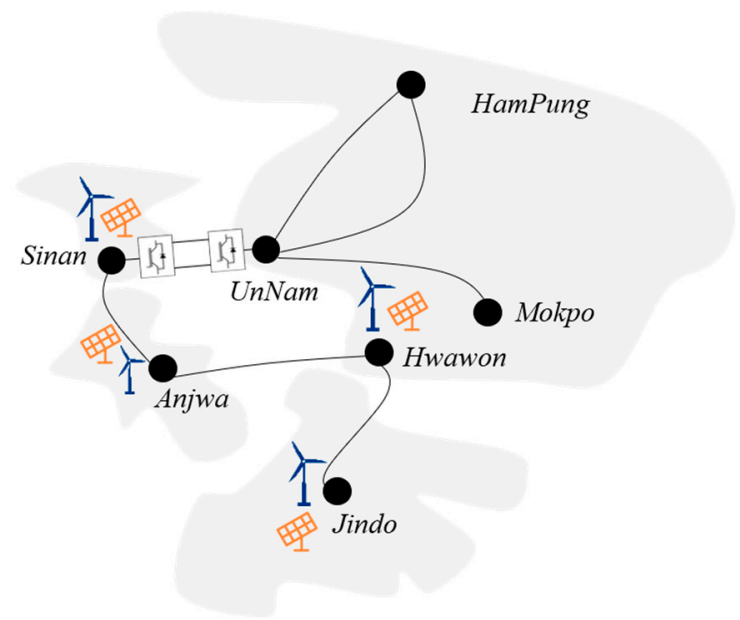

Figure 11. MVDC link candidate \#2. UnNam-Sinan connection.

The main purpose of the MVDC link as a second candidate is to enable efficient grid operation by minimizing the total line losses in a network with high penetration of REG. Therefore, the objective function for the optimization process is the sum of line loss $\left(f_{\text {loss }}\right)$ in Area 2, defined as (6). The maximum capacity of MVDC link is assumed to be 80 MVA.

Based on the total load demand and generation data in Area 2 during the day, which is shown in Figure 12, the optimal operating point of the MVDC link is determined hourly. The result is shown in Figure 13. The positive sign of $P_{\text {opt }}$ means that the MVDC link should transfer active power from 'Sinan' to 'UnNam1'.

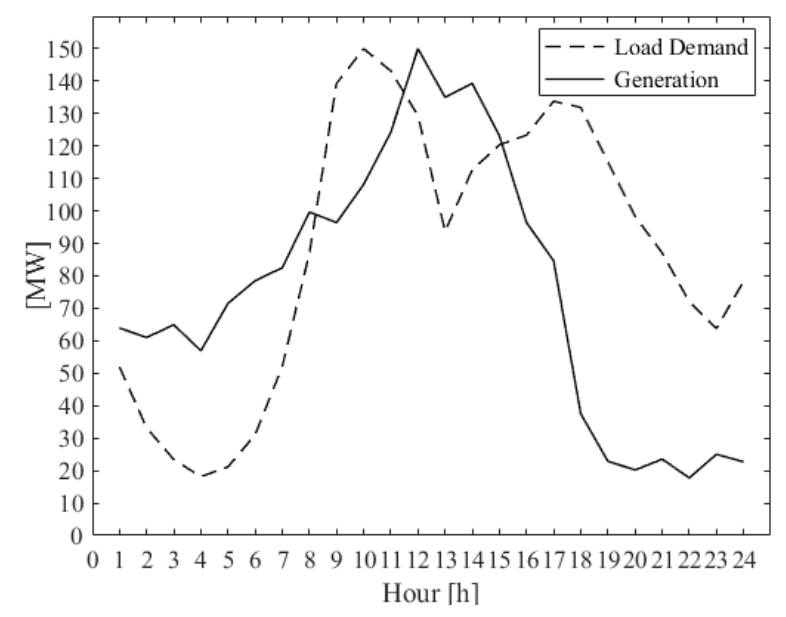

Figure 12. Daily profiles of total load and generation in Area 2.

Figure 14 shows the total line losses in Area 2 when the MVDC link is operated with the proposed scheme. In the case of the second candidate for the MVDC link, the sum of line loss in Area 2 over a day is about $12.4 \mathrm{MWh}$, which is about $43 \%$ less than total line losses in the existing AC grid (21.7 MWh). In other words, it can reduce total line losses of about 9.3 MWh per day compared to the existing AC grid by using the optimal operation through the MVDC link.

In the same way as the comparison in the MVDC link candidate \#1 case, we performed a comparison of the total line losses in operating the MVDC link as fixed active power setpoint of $60 \mathrm{MW}$ [29] with the proposed optimal operation. When the MVDC link is operated at a fixed output of 60MW for one day, the total line losses are about 13.6 MWh, which are about 8.1 MWh higher per day than when the optimal operation is performed. 


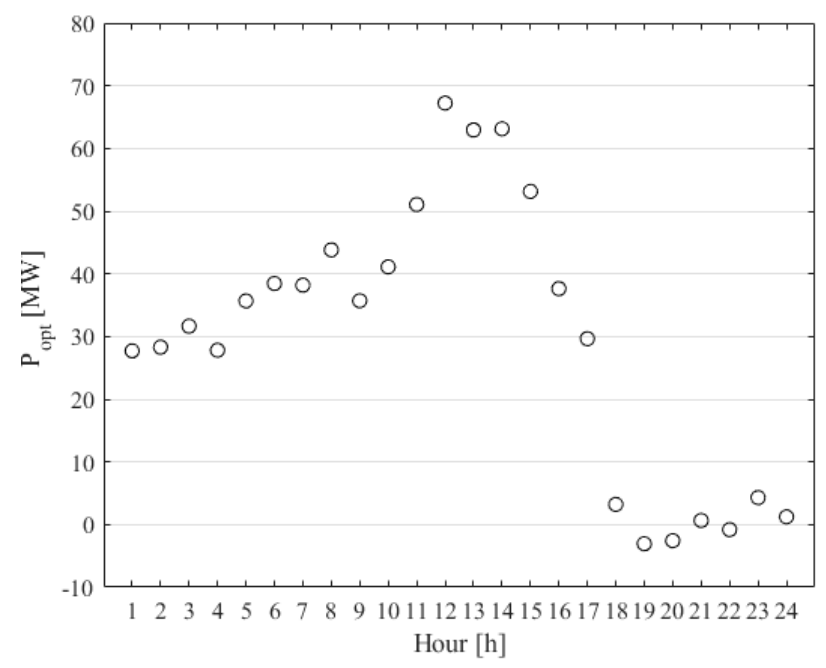

Figure 13. Optimal operating point of MVDC link candidate \#2.

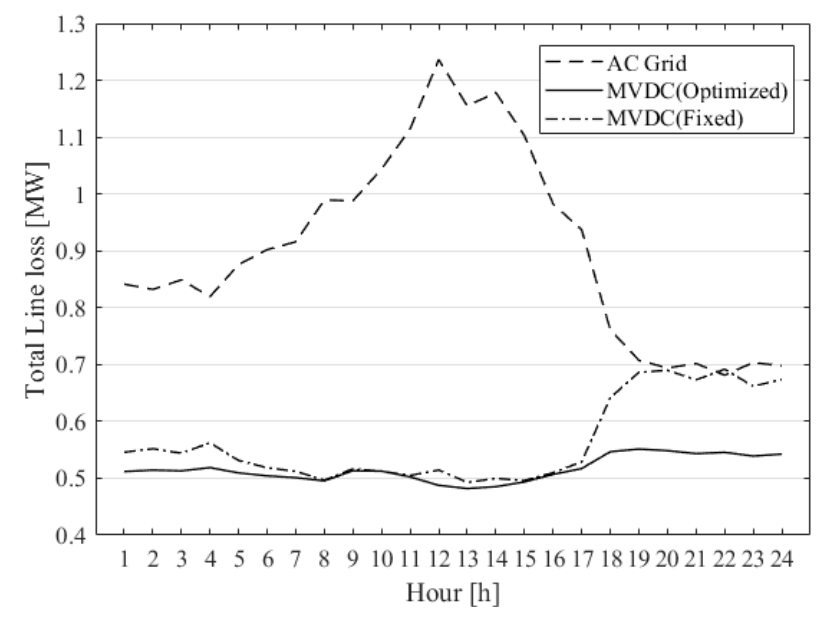

Figure 14. Comparison of total line losses in Area 2.

By controlling the MVDC link to minimize the total line losses in some areas, the network operator can economically operate the network in preparation for various load demand and generation. The economic benefits from reduced total line losses can be approximated using system marginal price (SMP) of the Korean electricity market, which means electricity import prices. Average annual SMP in 2016 is about $\$ 70 / \mathrm{MWh}$ in Korea [27]. The results show that $9.3 \mathrm{MWh}$ of daily loss can be reduced, while saving $\$ 651$ per day for the system operator if the MVDC link is operated with the proposed loss minimization mode.

Also, by comparing with the fixed power control mode, it is verified that through the optimal operation of the MVDC link, objective function defined by the system operator can be minimized every time. Since the optimal operating point calculated by the proposed method is the global minimum point with the lowest objective functions value, the amount of active power setpoint in the fixed power method is not important when making a comparison with the proposed method. Rather, it is clear that if the MVDC link is operated at other operating point which is not the optimal point, it is difficult to lower the value of the objective function. This means that the efficiency of the grid service desired by the system operator is decreased. In other words, the optimized operation of the MVDC link can provide a grid service that is most suitable to the system operator's purpose over time, using proposed method.

When determining the optimal operating points for the MVDC link, the objective function can be set differentially depending on the purpose of the network operator. In order to secure flexibility in the 
network and to prevent the effect of fluctuation of REG by reducing the variation of the line utilization rate in the networks, it is possible to set the object functions as $f_{L C U}$ and $f_{D E V}$. If the economically efficient network operation through line loss minimization is prioritized, the objective function can be set as total line losses $\left(f_{\text {loss }}\right)$. Because the output point of the MVDC link varies over time, there may be variations in the voltage magnitude of the buses in the area. Appendix B shows the voltage magnitude over time for all buses in the test system for each case study performed in this paper. Since the values of voltage limit are reflected as an inequality constraint in the optimization process, the voltage maintenance condition is not violated when the MVDC link is operated using the calculated operating points by the optimization process.

\subsection{Decentralized Emergency Operation of MVDC Link}

To validate the proposed DEC strategy, the control scheme is applied to the second candidate of the MVDC link and simulation studies are performed. Using the load and generation data for a day of Area 2, shown in Figure 12, the possible range of total amount of difference between generation and load demand in Area $2\left(P_{\text {Net-Area }}\right)$ is calculated. In order to improve the accuracy of the curve-fitting results, some margin was considered in the $P_{\text {Net-Area2 }}$ range and calculated to be from $-100 \mathrm{MW}$ to $40 \mathrm{MW}$.

Thereafter, a large number of power flow calculations are performed to record the voltage angle of PCC $\left(\theta_{P C C}\right)$ while changing the $P_{r e f}$ from $-100 \mathrm{MW}$ to $100 \mathrm{MW}$ step by step for every possible $P_{\text {Net-Area2 }}($ from $-100 \mathrm{MW}$ to $40 \mathrm{MW})$. The data obtained as a result of power flow calculation $\left(P_{\text {ref }}\right.$, $P_{\text {net-Area } 2}$, and $\left.\theta_{P C C}\right)$ is placed on the three-dimensional plot of the $\mathrm{x}-, \mathrm{y}^{-}$, and $\mathrm{z}$-axis. Then, the Curve Fitting progress [25] is performed to form an approximated surface as shown in Figure 15 and derive a linearized function as (22). $\alpha, \beta$ and $\gamma$ in (22) indicate the coefficients of each input variable calculated by using Curve Fitting progress.

$$
\begin{aligned}
& \theta_{P C C}=\alpha \cdot P_{r e f}+\beta \cdot P_{\text {Net-Area } 2}+\gamma \\
& P_{\text {Net-Area } 2}=a \cdot P_{r e f}+b \cdot \theta_{P C C}+c
\end{aligned}
$$

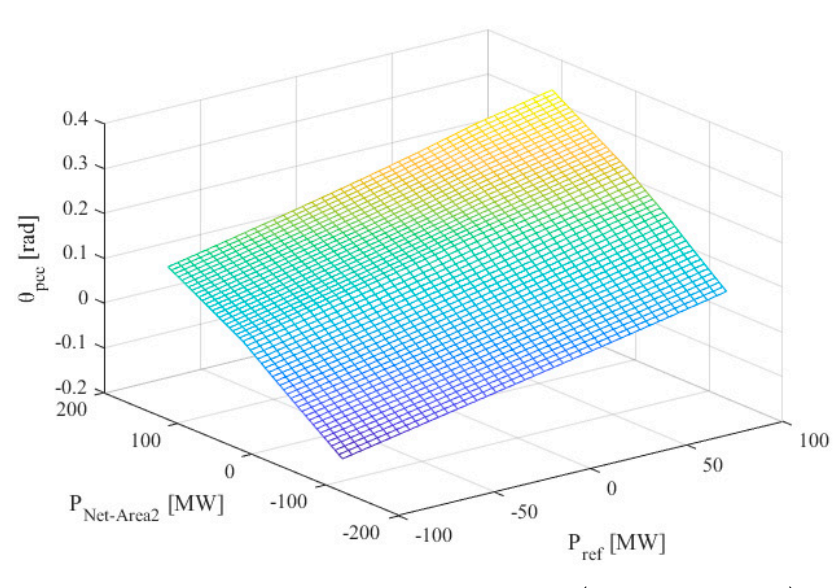

Figure 15. Response curve of $\theta_{P C C}=f\left(P_{\text {net-Area2 }}, P_{\text {ref }}\right)$.

Equation (22) can be expressed as (23) by changing the relation for $P_{\mathrm{Net}-\mathrm{Area2} .} a, b$ and $c$ in (23) are the coefficients of each variable. These can be calculated by rearranging (22). Table 1 shows the value of coefficients in (22) and (23) derived from this data build-up process for Area 2. 
Table 1. Parameters of response curves.

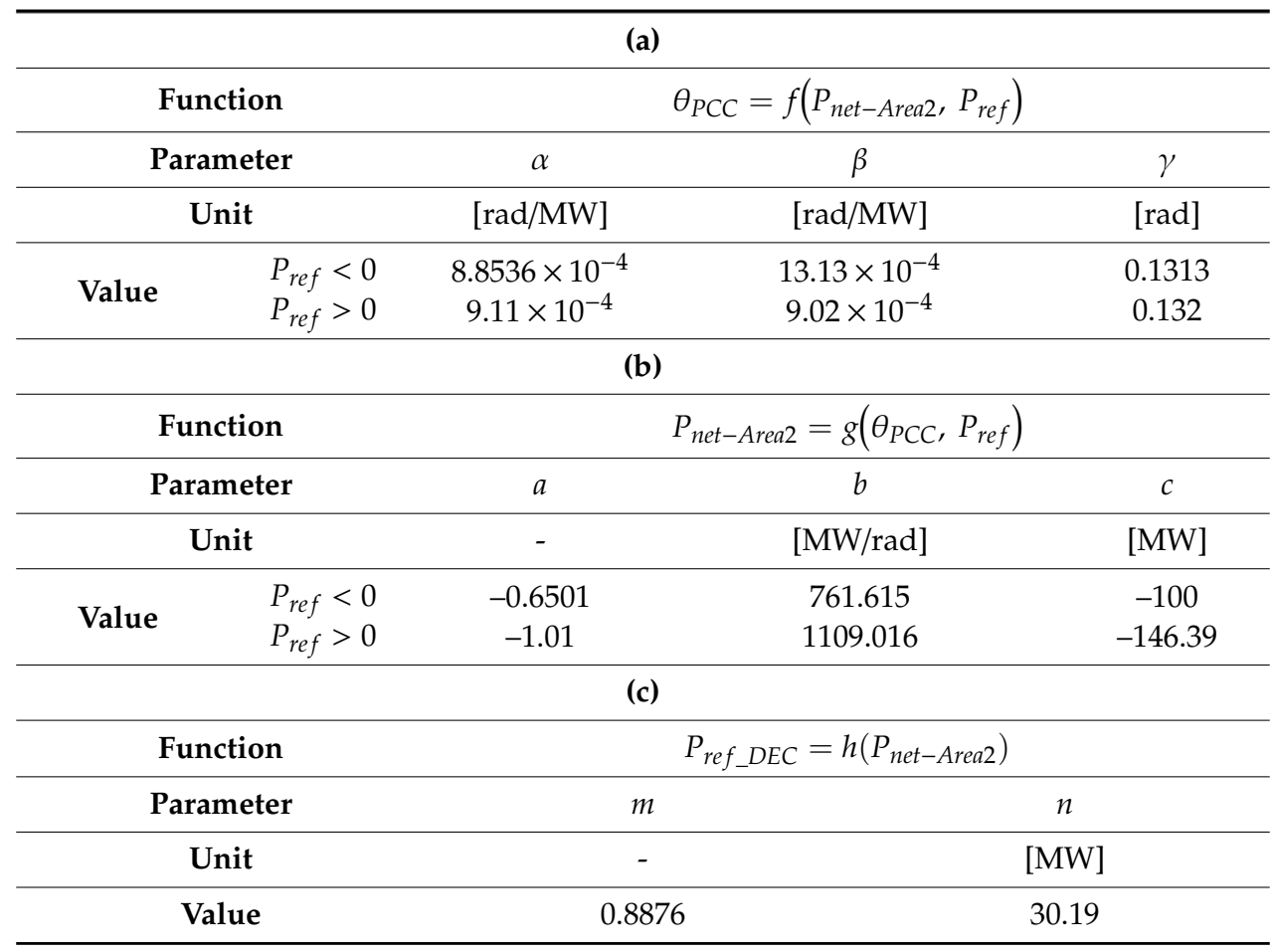

Observing the voltage angle of the bus and output power value of converter yields that the VSC station can calculate the total power generation and load difference in Area 2 by using the linearized response curve for $P_{\text {Net-Area2 }}$ shown in (23). However, $P_{\text {Net-Area } 2}$ indicates the difference between the total power generation and load demand amount of the area, and this is insufficient to calculate the optimum operating point by using the PSO algorithm.

Thus, in this paper, when calculating the operating point based on $P_{\text {Net-Area2 }}$, we use the AC transmission line emulation method, which imitates the power flow when the MVDC link is assumed to be an AC line, and determines it as a new operating point $\left(P_{r e f_{-} D E C}\right)$. The AC transmission line emulation method has been proposed in [30] for the purpose of solving the overload at a specific transmission line by making the DC line behave like the existing AC line in the network. The response curve of the power flow in the AC line according to $P_{\text {Net-Area2 }}$ is shown in Figure 16.

$$
P_{\text {ref_DEC }}=m \cdot P_{\text {Net-Area } 2}+n
$$

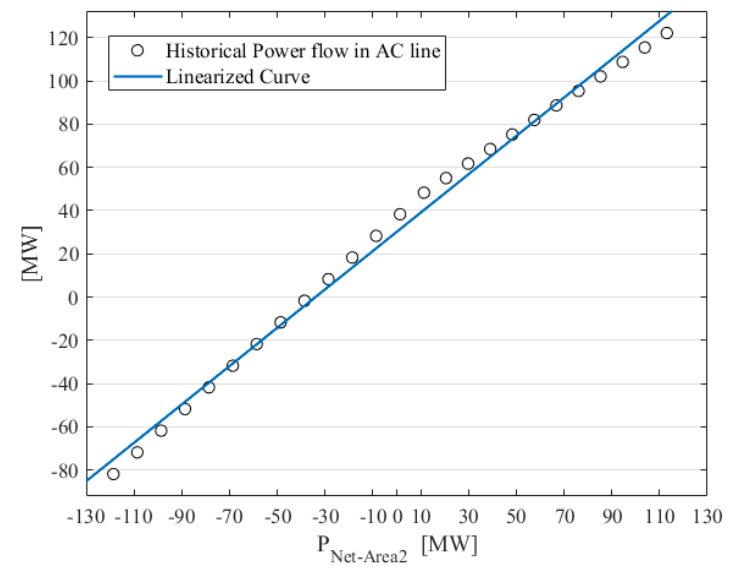

Figure 16. Response curve of $P_{\text {ref_DEC }}=h\left(P_{\text {net-Area2 }}\right)$. 
Then, through linearization, the formula is derived as (24). $m$ and $n$ are the coefficients calculated from the linearized response curve.

Using the response curves of Equations (22), (23) and (24), the VSC station can determine the emergency operating point by itself. Figure 17 shows the comparison of operating points of the MVDC link after the application of the proposed control method. The MVDC link was operated to minimize total line losses as in Figure 13, and it is assumed that a communication failure occurred at Hour $=12 \mathrm{~h}$.

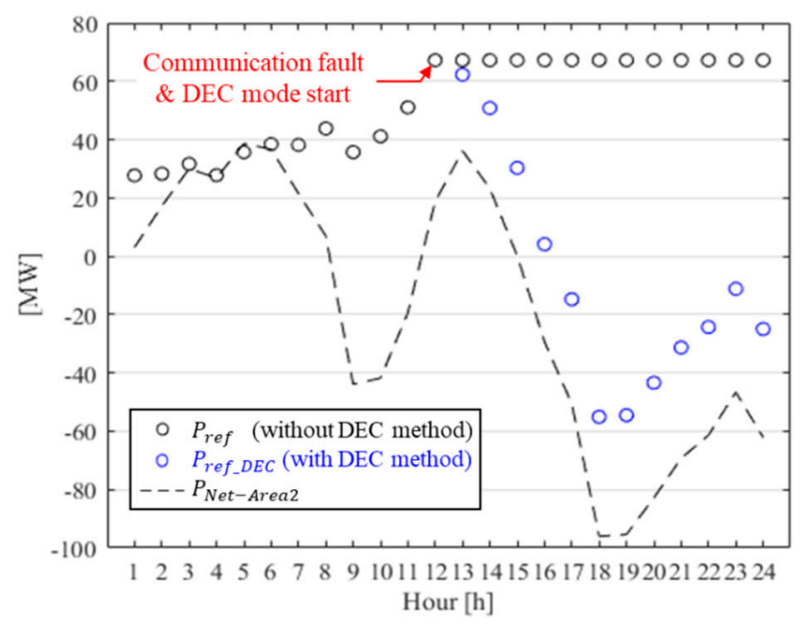

Figure 17. Comparison of operating points in two control mode.

If the proposed emergency control is not applied, the operation point of VSC station cannot be changed after a communication failure. When the proposed method is applied, after detecting communication failure, the VSC station can change the operating mode and calculate a new operating point by itself in accordance with the state of the connected network. However, this method is not the most optimal operation point, because it is a temporary control method in on-line operation for considering only the total power generation amount and load demand of the connected grid. Therefore, further study into the algorithm that can calculate the most optimal operating point in real time by only using the information of $P_{\text {Net-Area }}$ calculated by VSC station through (23) is needed.

\section{Conclusions}

Through controlling the power flow using a DC line at the intermediate voltage level of the transmission and distribution network, it is possible to maximize the utilization of the transmission line and minimize total system line loss. In addition, it was verified in this paper that to reflect the variation of the REG with time, the optimal operating point of the MVDC link can be determined using a multi-objective optimization algorithm in which objective functions such as minimizing the system loss and leveling the line utilization are included. The main purpose of the system operator when operating the MVDC link to enhance the transmission line capacity utilization is to make the power grid more flexible and to reduce the expansion of new substations and lines, which are overloaded.

Thus, it is difficult to calculate the exact economic benefit for this. However, if the MVDC link is operated to minimize the total transmission line loss; then, the economical benefits using Korea SMP can be estimated. Comparing to the usage of the AC line, it is verified that the line loss of $9.3 \mathrm{MWh}$ per day can be reduced so that it could achieve an economic benefit of about $\$ 651$ per day.

If the VSC station connected to the DC line can detect the difference between the total power generation and the load amount of the network, the new operating point can be calculated reflecting the variation of REG without needing communication with the system operator. However, the strategy of calculating the optimal operating point for the purpose of operating by only using information regarding the difference between total generation and load requires further investigation in the future. 
Author Contributions: The main idea was proposed by C.H., S.S., J.K. and G.J. The operating strategy and optimization algorithm were proposed by C.H. and S.S. The experimental results were collected and analyzed by C.H.

Funding: This research received no external funding.

Acknowledgments: This work was supported by National Research Foundation of Korea (No.2017K1A4A3013579) and "Human Resources Program in Energy Technology" of the Korea Institute of Energy Technology Evaluation and Planning (KETEP), granted financial resource from the Ministry of Trade, Industry and Energy, Republic of Korea (No.20174030201540).

Conflicts of Interest: The authors declare no conflict of interest.

\section{Nomenclature}

$n_{l}, n_{B} \quad$ Total number of branches and buses in network.

$n_{G}, n_{L} \quad$ Total number of generators and loads in network.

$P_{V S C_{k}}, Q_{V S C_{k}} \quad$ Active and reactive power injected into the network from $k$ th VSC.

$Q_{\text {min }_{k}}, Q_{\text {max }_{k}} \quad$ Lower and upper limit of reactive power output of $k$ th VSC.

$S_{V S C_{k}}$

$P_{G(i)}$

$P_{L(i)}$

$P_{\text {net-Area }}$

$P_{\text {ref }}$

$P_{\text {ref_DEC }}$

$P_{\text {opt }}$

Apparent power injected into the network from $k$ th VSC.

Active power generated by $i$ th generator.

Active power consumed by $i$ th load.

Total amount of difference between generation and load demand in the area.

Active power reference of MVDC link.

Active power reference of MVDC link with DEC.

$I_{i} \quad$ Current magnitude of $i$ th branch.

$r_{i} \quad$ Resistance of $i$ th branch.

$S_{i} \quad$ Apparent power flow of $i$ th branch.

$S_{i, \text { rate }} \quad$ Rated apparent power of $i$ th branch

$S_{i, \max } \quad$ Upper limit of apparent power of $i$ th branch.

$V_{k}, \theta_{k} \quad$ Voltage magnitude and angle of $k$ th bus.

$V_{\text {min }}, V_{\max } \quad$ Lower and upper limit of voltage magnitude of $k$ th bus.

$n_{P} \quad$ Total number of particles.

$n_{o b j} \quad$ Total number of objective functions.

$n_{\text {opt }} \quad$ Total number of particles on pareto optimal front.

$x_{k} \quad$ Position of $k$ th particle on pareto optimal front.

$x_{\text {opt }} \quad$ Position of particle calculated as compromise solution.

$x_{n}(t) \quad$ Position of $n$th particle at iteration step $t$

$p_{n}(t) \quad$ Best positions of $n$th particle among its previously iterative path at iteration step $t$.

$g(t) \quad$ Global optimum position at iteration step $t$.

$v_{n}(t) \quad$ Velocity of $n$th particle at iteration step $t$.

$w, c_{1}, c_{2} \quad$ Weight factors for each term in the particle's velocity equation.

\section{Appendix A}

In this chapter, detailed information of the test system used in the case study is presented. Table A1 shows the numbering of the AC lines of the test system shown in Figure 3. Table A1 (a) shows the branch numbers from 1 of 7 , and (b) is from 8 to 13 . This is to provide an understanding of the branch number shown in Figure 10. 
Table A1. Branch number information.

\begin{tabular}{|c|c|c|c|c|c|c|c|}
\hline \multicolumn{4}{|c|}{ (a) } & \multicolumn{4}{|c|}{ (b) } \\
\hline \multirow{2}{*}{$\begin{array}{l}\text { Branch } \\
\text { Number }\end{array}$} & \multicolumn{2}{|c|}{ Bus Name } & \multirow{2}{*}{$\begin{array}{c}\text { Branch } \\
\text { ID }\end{array}$} & \multirow{2}{*}{$\begin{array}{l}\text { Branch } \\
\text { Number }\end{array}$} & \multicolumn{2}{|c|}{ Bus Name } & \multirow{2}{*}{$\begin{array}{c}\text { Branch } \\
\text { ID }\end{array}$} \\
\hline & From & To & & & From & To & \\
\hline 1 & UnNam1 & Sinan & 1 & 8 & HamPung1 & SujunNam & 2 \\
\hline 2 & UnNam1 & Sinan & 2 & 9 & Umda & Naju & 1 \\
\hline 3 & UnNam1 & HamPung1 & 1 & 10 & Umda & Naju & 2 \\
\hline 4 & UnNam1 & HamPung1 & 2 & 11 & Umda & Mokpo & 1 \\
\hline 5 & UnNam1 & Umda & 1 & 12 & Umda & Mokpo & 2 \\
\hline 6 & UnNam1 & Umda & 2 & 13 & UnNam2 & HamPung2 & 1 \\
\hline 7 & HamPung1 & SujunNam & 1 & 14 & UnNam2 & HamPung2 & 2 \\
\hline
\end{tabular}

Table A2. Renewable energy generation (REG) and load capacity information.

\begin{tabular}{|c|c|c|c|c|c|c|c|}
\hline \multirow{4}{*}{ Area Number } & \multirow{4}{*}{ Bus Name } & \multicolumn{6}{|c|}{ Maximum Capacity } \\
\hline & & \multicolumn{4}{|c|}{ REG } & \multirow{2}{*}{\multicolumn{2}{|c|}{ Load }} \\
\hline & & \multicolumn{2}{|c|}{ PV } & \multicolumn{2}{|c|}{$\begin{array}{l}\text { Wind } \\
\text { Turbine }\end{array}$} & & \\
\hline & & $\begin{array}{c}\mathbf{P}_{\max } \\
{[\mathbf{M W}]}\end{array}$ & $\begin{array}{l}\text { Power } \\
\text { Factor }\end{array}$ & $\begin{array}{c}\mathbf{P}_{\max } \\
\text { [MW] }\end{array}$ & $\begin{array}{l}\text { Power } \\
\text { Factor }\end{array}$ & $\begin{array}{l}\mathbf{P}_{\max } \\
{[\mathbf{M W}]}\end{array}$ & $\begin{array}{c}\mathbf{Q}_{\max } \\
\text { [MVar] }\end{array}$ \\
\hline \multirow{9}{*}{ Area1 } & SujunNam & - & - & - & - & - & - \\
\hline & HamPung1 & - & - & - & - & 7.0 & 2.5 \\
\hline & HamPung2 & - & - & - & - & - & - \\
\hline & UnNam1 & 50.3 & 1.0 & 37.1 & 0.95 & 9.5 & 3.4 \\
\hline & UnNam2 & - & - & - & - & - & - \\
\hline & Umda & 22.6 & 1.0 & - & - & 35.4 & 12.7 \\
\hline & Mokpo & - & - & - & - & 49.9 & 17.9 \\
\hline & Naju & - & - & - & - & 48.2 & 17.3 \\
\hline & Total & 72.9 & - & 37.1 & - & 150 & 53.8 \\
\hline \multirow{7}{*}{ Area2 } & Sinan & 12.0 & 1.0 & 19.8 & 0.95 & - & - \\
\hline & Anjwa1 & 27.5 & 1.0 & 23.0 & 0.95 & 6.7 & 2.4 \\
\hline & Anjwa2 & - & - & - & - & - & - \\
\hline & Hwawon & 25.8 & 1.0 & 1.1 & 0.95 & 54.2 & 19.5 \\
\hline & Jindo & 6.6 & 1.0 & 34.2 & 0.95 & 12.0 & 4.3 \\
\hline & Jindo CS & - & - & - & - & 77.1 & 27.7 \\
\hline & Total & 71.9 & - & 78.1 & - & 150 & 53.9 \\
\hline
\end{tabular}

Table A2 shows the information about maximum power capacity of the REG and load demand of the test system, which is used in the case study. This information is based on real data from the Korean power system planning in 2031. Maximum capacity means the value that represents maximum power generation and maximum load demand in one day, respectively, as shown in Figure 5 and 12. It is assumed that the PV generator in Table A2 has a fixed power factor of 1.0 and the wind turbine has 0.95 .

\section{Appendix B}

In the power transmission system of South Korea, the voltage magnitude maintenance standard for steady-state stability is shown in the Table A3 [31].

Table A3. Voltage maintenance standard in the Korean power system.

\begin{tabular}{cccc}
\hline Rated Voltage & $\begin{array}{c}\text { Maintenance } \\
\text { Range }\end{array}$ & $\begin{array}{c}\text { Minimum Limit } \\
\left(\boldsymbol{V}_{\text {min }}\right)\end{array}$ & $\begin{array}{c}\text { Maximum Limit } \\
\left(\boldsymbol{V}_{\text {max }}\right)\end{array}$ \\
\hline $765 \mathrm{kV}$ & $\pm 5 \%$ & $0.95 \mathrm{p} \cdot \mathrm{u}$ & $1.05 \mathrm{p} . \mathrm{u}$ \\
$345 \mathrm{kV}$ & $\pm 5 \%$ & $0.95 \mathrm{p} \cdot \mathrm{u}$ & $1.05 \mathrm{p} \cdot \mathrm{u}$ \\
$154 \mathrm{kV}$ & $\pm 10 \%$ & $0.90 \mathrm{p} \cdot \mathrm{u}$ & $1.10 \mathrm{p} \cdot \mathrm{u}$ \\
\hline
\end{tabular}

Since case studies in this paper were conducted in a $154 \mathrm{kV}$ transmission network, the voltage maintenance range of $\pm 10 \%$ of rated voltage was applied as an inequality constraint in optimization process to calculate the optimal operating points of the MVDC link. 


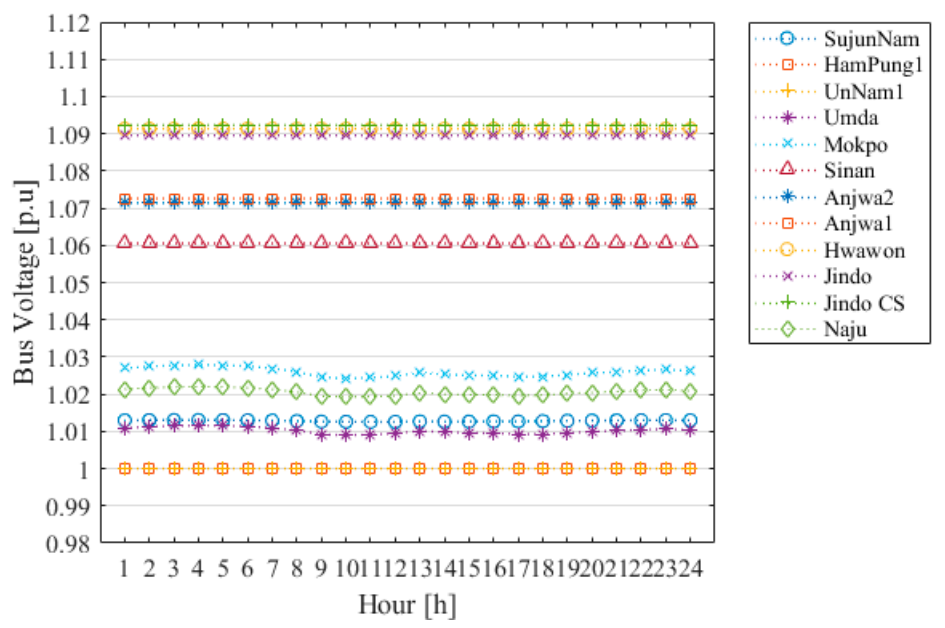

Figure A1. Voltage profile for case study of MVDC candidate \#1.

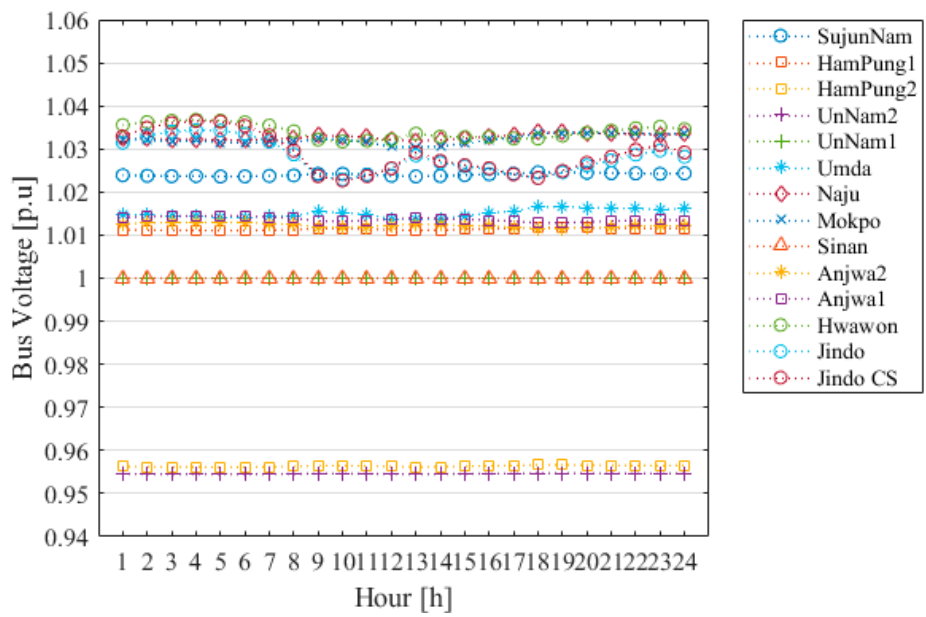

Figure A2. Voltage profile for case study of MVDC candidate \#2.

Figures A1 and A2 show the voltage magnitude over time for all buses in the test system of each case study performed in this paper. Since two converters of the MVDC link perform the AC voltage control, the voltage magnitudes at the PCC of the converter is 1.0 p.u and the voltage magnitudes at the other buses are also within the maintenance range (from 0.90 to 1.10 p.u).

\section{References}

1. Agency, I.E. World Energy Outlook 2018. Available online: https://doi.org/10.1787/weo-2018-en (accessed on 13 November 2018).

2. Walling, R.A.; Saint, R.; Dugan, R.C.; Burke, J.; Kojovic, L.A. Summary of Distributed Resources Impact on Power Delivery Systems. IEEE Trans. Power Deliv. 2008, 23, 1636-1644. [CrossRef]

3. Sun, S.; Dong, M.; Liang, B. Distributed Real-Time Power Balancing in Renewable-Integrated Power Grids with Storage and Flexible Loads. IEEE Trans. Smart Grid 2016, 7, 2337-2349. [CrossRef]

4. Sgouras, K.I.; Bouhouras, A.S.; Gkaidatzis, P.A.; Doukas, D.I.; Labridis, D.P. Impact of reverse power flow on the optimal distributed generation placement problem. IET Gener. Transm. Distrib. 2017, 11, 4626-4632. [CrossRef]

5. Usaola, J. Probabilistic load flow with wind production uncertainty using cumulants and Cornish-Fisher expansion. Int. J. Electr. Power E Energy Syst. 2009, 31, 474-481.

6. Fan, M.; Vittal, V.; Heydt, G.T.; Ayyanar, R. Probabilistic Power Flow Analysis with Generation Dispatch Including Photovoltaic Resources. IEEE Trans. Power Syst. 2013, 28, 1797-1805. [CrossRef]

7. Sciano, D.; Raza, A.; Salcedo, R.; Diaz-Aguilo, M.; Uosef, R.E.; Czarkowski, D.; de Leon, F. Evaluation of DC Links on Dense-Load Urban Distribution Networks. IEEE Trans. Power Deliv. 2016, 31, 1317-1326. [CrossRef] 
8. Cao, W.; Wu, J.; Jenkins, N. Feeder load balancing in MV distribution networks using soft normally-open points. In Proceedings of the IEEE PES Innovative Smart Grid Technologies, Europe, Istanbul, Turkey, 12-15 October 2014; pp. 1-6.

9. Bloemink, J.M.; Green, T.C. Increasing distributed generation penetration using soft normally-open points. In Proceedings of the IEEE PES General Meeting, Providence, RI, USA, 25-29 July 2010; pp. 1-8.

10. Qi, Q.; Wu, J.; Long, C. Multi-objective operation optimization of an electrical distribution network with soft open point. App. Energy 2017, 208, 734-744. [CrossRef]

11. Zhang, L.; Liang, J.; Tang, W.; Li, G.; Cai, Y.; Sheng, W. Converting AC Converting AC distribution lines to DC to increase transfer capacities and DG penetration. IEEE Trans. Smart Grid 2018. [CrossRef]

12. Qi, Q.; Long, C.; Wu, J.; Yu, J. Impacts of a medium voltage direct current link on the performance of electrical distribution networks. App. Energy 2018, 230, 175-188. [CrossRef]

13. Frey, K.; Wiest, P.; Rudion, K.; Christian, J. Automated operation of parallel VSC HVDC links within an interconnected AC network. In Proceedings of the 2016 IEEE Power and Energy Society General Meeting (PESGM), Boston, MA, USA, 17-21 July 2016; pp. 1-5.

14. Abido, M.A. Multiobjective particle swarm optimization for environmental/economic dispatch problem. Electr. Power Syst. Research 2009, 79, 1105-1113. [CrossRef]

15. Kennedy, J.; Eberhart, R.C. Particle swarm optimization. In Proceedings of the IEEE International Conference on Neural Networks, Perth, WA, Australia, 27 November-1 December 1995; Volume 4, pp. 1942-1948.

16. Reyes-Sierra, M.; Coello, C.A.C. Multi-Objective Particle Swarm Optimizers: A Survey of the State-of-the-Art. Int. J. Comput. Intel. Res. 2006, 2, 287-308.

17. Urquidez, O.A.; Xie, L. Singular value sensitivity based optimal control of embedded VSC-HVDC for steady-state voltage stability enhancement. IEEE Trans. Power Syst. 2016, 31, 216-225. [CrossRef]

18. Feng, W.; Tjernberg, L.B.; Mannikoff, A.; Bergman, A. A new approach for benefit evaluation of multiterminal VSC-HVDC using a proposed mixed AC/DC optimal power flow. IEEE Trans. Power Deliv. 2014, 29, 432-443. [CrossRef]

19. Guo, Y.; Gao, H.; Wu, Q.; Zhao, H.; Østergaard, J.; Shahidehpour, M. Enhanced voltage control of VSC-HVDC-connected offshore wind farms based on model predictive control. IEEE Trans. Sustain. Energy 2018, 9, 474-487. [CrossRef]

20. Zamani, M.A.; Yazdani, A.; Sidhu, T.S. A Communication-Assisted Protection Strategy for Inverter-Based Medium-Voltage Microgrids. IEEE Trans. Smart Grid 2012, 3, 2088-2099. [CrossRef]

21. Fazeli, A.; Sumner, M.; Johnson, M.C.; Christopher, E. Real-time deterministic power flow control through dispatch of distributed energy resources. IET Gener. Transm. Distrib. 2015, 9, 2724-2735. [CrossRef]

22. Qi, C.; Wang, K.; Fu, Y.; Li, G.; Han, B.; Huang, R.; Pu, T. A Decentralized Optimal Operation of AC/DC Hybrid Distribution Grids. IEEE Trans. Smart Grid 2018, 9, 6095-6105. [CrossRef]

23. Calderaro, V.; Conio, G.; Galdi, V.; Massa, G.; Piccolo, A. Optimal Decentralized Voltage Control for Distribution Systems with Inverter-Based Distributed Generators. IEEE Trans. Power Systems 2014, 29, 230-241. [CrossRef]

24. Baumgartner, U.; Magele, C.; Renhart, W. Pareto Optimality and Particle Swarm Optimization. IEEE Trans. Magnetics 2004, 40, 1172-1175. [CrossRef]

25. MATLAB Curve Fitting Toolbox: User's Guide. Available online: https://www.mathworks.com/help/pdf doc/curvefit/curvefit.pdf (accessed on March 2019).

26. Oh, S.; Shin, H.; Cho, H.; Lee, B. Transient Impact Analysis of High Renewable Energy Sources Penetration According to the Future Korean Power Grid Scenario. Sustainability 2018, 10, 4140. [CrossRef]

27. Kim, S.; Lee, H.; Kim, H.; Jang, D.-H.; Kim, H.-J.; Hur, J.; Cho, Y.-S.; Hur, K. Improvement in policy and proactive interconnection procedure for renewable energy expansion in South Korea. Renew. Sustain. Energy Rev. 2018, 98, 150-162. [CrossRef]

28. Zavala, V.M.; Flores-Tlacuahuac, A. Stability of multiobjective predictive control: A utopia-tracking approach. Automatica 2012, 48, 2627-2632. [CrossRef]

29. Mohammadi, F. Power Management Strategy in Multi-Terminal VSC-HVDC System. In Proceedings of the 4th National Conference on Applied Research in Electrical, Mechanical, Computer and IT Engineering, Tehran, Iran, 4 October 2018. 
30. Song, S.; Kim, J.; Lee, J.; Jang, G. AC Transmission Emulation Control Strategies for the BTB VSC HVDC System in the Metropolitan Area of Seoul. Energies 2017, 10, 1143. [CrossRef]

31. Kang, H.-K.; Kim, T.-E.; Kim, K.-H.; Choi, Y.-M.; Yu, H.-J.; Lee, G.-W.; Song, S.-H. Optimal power system operation by EMS and MOS in KPX. In Proceedings of the 2009 Transmission \& Distribution Conference \& Exposition: Asia and Pacific, Seoul, Korea, 26-30 October 2009; pp. 1-5. 